\title{
Genome-wide comparative analysis of metacaspases in unicellular and filamentous cyanobacteria
}

\author{
Qiao Jiang ${ }^{1}$, Song Qin², Qing-yu Wu ${ }^{1 *}$
}

\begin{abstract}
Background: Cyanobacteria are an ancient group of photoautotrophic prokaryotes with wide variations in genome size and ecological habitat. Metacaspases (MCAs) are cysteine proteinases that have sequence homology to caspases and play essential roles in programmed cell death (PCD). MCAs have been identified in several prokaryotes, fungi and plants; however, knowledge about cyanobacterial metacaspases still remains obscure. With the availability of sequenced genomes of 33 cyanobacteria, we perform a comparative analysis of metacaspases and explore their distribution, domain structure and evolution.

Results: A total of 58 putative MCAs were identified, which are abundant in filamentous diazotrophic cyanobacteria and Acaryochloris marina MBIC 11017 and absent in all Prochlorococcus and marine Synechococcus strains, except Synechococcus sp. PCC 7002. The Cys-His dyad of caspase superfamily is conserved, while mutations (Tyr in place of His and Ser/Asn/Gln/Gly instead of Cys) are also detected in some cyanobacteria. MCAs can be classified into two major families ( $\alpha$ and $\beta$ ) based on the additional domain structure. Ten types and a total of 276 additional domains were identified, most of which involves in signal transduction. Apoptotic related NACHT domain was also found in two cyanobacterial MCAs. Phylogenetic tree of MCA catalytic P20 domains coincides well with the domain structure and the phylogenies based on 16s rRNA.

Conclusions: The existence and quantity of MCA genes in unicellular and filamentous cyanobacteria are a function of the genome size and ecological habitat. MCAs of family $\alpha$ and $\beta$ seem to evolve separately and the recruitment of WD40 additional domain occurs later than the divergence of the two families. In this study, a general framework of sequence-structure-function connections for the metacaspases has been revealed, which may provide new targets for function investigation.
\end{abstract}

\section{Background}

Cyanobacteria are among the earliest branching groups on earth, dating back 2.5-3.5 billion years, based on the fossil evidence [1]. As a taxonomic unit characterized by the ability to execute oxygenic photosynthesis, cyanobacteria constitute a group of species diverse in genome size and ecological habitats, indicating the significance of comparative genome research. Cyanobacteria, with a variation in genome size from 1.6 Mb (Prochlorococcus sp. MIT9301) to $9.2 \mathrm{Mb}$ (Nostoc punctiforme PCC 73102), are found in almost every imaginable environment, from ocean to fresh water to bare rock. Cyanobacteria also inhabit in the

\footnotetext{
* Correspondence: qingyu@mails.tsinghua.edu.cn

${ }^{1}$ School of Life Sciences, Tsinghua University, Beijing, 100084, China
}

extreme environments, for example, Synechococcus sp. JA-2-3B'a (2-13) and Synechococcus sp. JA-3-3Ab were separated from hot spring. As unicellular and nonnitrogen-fixing cyanobacteria, Prochlorococcus sp. and Synechococcus sp. maintain the smallest genome sizes and account for significant biomass and primary production of marine biosphere [2]. Other unicellular species have larger genome sizes, including water bloom forming cyanobacteria (Synechocystis sp. PCC 6803 and Microcystis aeruginosa NIES-843), a thylakoids absence cyanobacterium (Gloeobacter sp. PCC 7421), a nitrogen-fixing cyanobacterium (Cyanothece sp. ATCC 51142), and an animal-cyanobacterial symbionsis (Acaryochloris marina MBIC11017). While the diazotrophic filamentous cyanobacteria comprise the largest genome size, such as Nostoc
Ciomed Central 
sp. PCC 7120, Anabaena variabilis ATCC 29413, plantcyanobacteria symbionsis Nostoc punctiforme PCC 73102 and marine Trichodesmium sp. IMS 101.

Programmed cell death (PCD) is a suicide mechanism to promote and maintain genetic stability [3]. PCD was considered as a characteristic of metazoans for a long time before apoptosis markers were found in yeast which indicates multicellularity is not the most important prerequisite[4]. Recently, PCD mechanism has been observed in all but one of the six/eight major groups of prokaryotes, with the exception of the rhizaria [5]. Experimental evidences for PCD in cyanobacteria come from three species, including the freshwater cyanobacterium Anabaena spp. exposed to univalent-cation salts, the bloom-causing cyanobacterium Microcystis aeruginosa from St. Lucie Estuary by treatment with $\mathrm{H}_{2} \mathrm{O}_{2}$ and Trichodesmium sp. IMS 101 suffering iron starvation and light irradiance [6-8].

Caspases (cysteinyl aspartate-specific proteases) are one of the most important and widely researched apoptotic proteins in mammalian PCD. Caspase was initially thought to be limited to metazoans, and no one had managed to identify caspase homologues, either in plants or bacteria. Then Uren and his colleagues identified two ancient families of caspase-like proteins, paracaspases and metacaspases in silico [9] and Khan and his co-workers demonstrated that a yeast metacaspase (YCA1) mediates PCD in Saccharomyces cerevisiae[10]. Hereafter, metacaspases were found involved in PCD of yeasts, filamentous fungi, plants, and a variety of bacteria. Most of these metacaspases share sequence homology with caspases, but show different substrate specificity [11-16]. Metacaspases belong to caspase family (C14), which are part of the clan CD, a family of proteases characteristic with their His/Cys catalytic dyad [17]. Metacaspases process a conserved caspase catalytic subunit P20 domain (COG 4249, KOG1546 in the NCBI Conserved Domain Database), and share conserved amino acid residues within His- and Cys-catalytic sites [18]. Interestingly, most of the typical genes encoding in metazoan PCD are missing in bacteria and early-branching eukaryotes, such as CAD and P53[19]. Therefore, the presence of metacaspases suggests a concernful role within PCD evolution.

Cyanobacteria maintain a rich metacaspase pool, and many of these genes have been identified in silico [20] was identified in some sequenced cyanobacteria strains, including Gloeobacter violaceus PCC 7421, Thermosynechococcus elongatus BP-1, Synechocystis sp. PCC 6803, Trichodesmium erythraeum ISM 101, Nostoc punctiforme PCC 73102, Nostoc sp. PCC 7120, and Anabaena variabilis ATCC 29413. Metacaspases were absent in MED3, Prochlorococcus marinus MIT 9313, SS 120 (CCMP 1375), Synechococcus sp. WH 8102, Synechococcus elongatus PCC 7942 and Synechococcus elongates [20]. With the completion of genome sequencing of several cyanobacterial species, modifications and supplements are needed.

As of November 2008, 33 genomes of unicellular and filamentous cyanobacteria became available, which facilitates cyanobacterial systemic analysis for restrictionmodification systems and serine/threonine protein kinases [21]. The comparative genome research on metacaspases and other PCD proteins in filamentous fungi has been documented [22]. Besides, Koonin and Aravind described a clear affinity of bacterial metacaspases and the metazoan caspases by phylogenetic analysis of caspase-like protease superfamily [19]. In this study, we selected five proven metacaspases in marine diatom Thalassiosira pseudonana to search for cyanobacterial metacaspases [11]. Metacaspases in Thalassiosira pseudonana were chosen due to the few metacaspases verified experimentally in cyanobacteria and the close evolutional relationship between cyanobacteria and eukaryotic phytoplankton. We employed a BLASTp-plus-phylogeny reconstruction approach [22] to analyze metacaspase sequences in cyanobacteria, and present an overall view of their classifications, structure, phylogeny and evolution. Better understanding of cyanobacterial metacaspases may provide further insights into evolution of PCD.

\section{Results}

\section{Identification of metacaspase proteins}

The 33 complete cyanobacterial genomes downloaded from IMG database were used in this research (Table 1, Figure 1). To identify proteins similar to proven metacaspases from Thalassiosira pseudonana, we performed BLASTp searches of the 33 cyanobacterial genomes. CDD [23,24] and SMART [25] analyses with the derived sequences were then carried out to eliminate false positives. Two proteins annotated as caspase catalytic subunit P20 in IMG database, including 638107126 (IMG Gene Object Identifier) from Trichodesmium erythraeum ISM 101 and 641540115 from Microcystis aeruginosa NIES-843, were found to lack catalytic domain and excluded. Of two proteins (637313946 from Thermosynechococcus elongatus BP-1, and 638107265 from Trichodesmium erythraeum ISM 101), CASc domain (caspase catalytic subunit P20) recognized by CDD was not identified in SMART analysis [23], and both of the proteins were excluded. Altogether, 58 putative metacaspase sequences were considered in this study (Table 2). Twenty-six of which were originally annotated as peptidase C14, caspase catalytic subunit p20 or protein containing caspase domain. The remaining 32 proteins were accepted as metacaspases in this research, including 18 proteins annotated by other additional domains (such as ATPase, GUN4-like family protein, Chase2 sensor protein and WD40 repeats), 12 proteins annotated as 
Table 1 Sequenced cyanobacterial strains and MCA information

\begin{tabular}{|c|c|c|c|c|c|}
\hline Strain & Key Feature & $\begin{array}{l}\text { Total } \\
\text { Proteins }\end{array}$ & $\begin{array}{l}\text { Total MCAs \& } \\
\text { Percentage }\end{array}$ & $\begin{array}{l}\mathrm{H} / \mathrm{C} \\
\text { Sites }\end{array}$ & $\begin{array}{l}\text { Additional } \\
\text { Domains }\end{array}$ \\
\hline Prochlorococcus marinus str. MIT 9215 & Unicellular Marine & 1983 & - & - & - \\
\hline Prochlorococcus marinus str. MIT 9301 & Unicellular Marine & 1907 & - & - & - \\
\hline Prochlorococcus marinus str. AS 9601 & Unicellular Marine & 1921 & - & - & - \\
\hline Prochlorococcus marinus str. MIT 9312 & Unicellular Marine & 1810 & - & - & - \\
\hline $\begin{array}{l}\text { Prochlorococcus marinus subsp. pastoris str. } \\
\text { CCMP } 1986\end{array}$ & Unicellular Marine & 1717 & - & - & - \\
\hline Prochlorococcus marinus str. MIT 9515 & Unicellular Marine & 1906 & - & - & - \\
\hline Prochlorococcus marinus str. NATL2A & Unicellular Marine & 2163 & - & - & - \\
\hline Prochlorococcus marinus str. NATL1A & Unicellular Marine & 2193 & - & - & - \\
\hline $\begin{array}{l}\text { Prochlorococcus marinus subsp. marinus str. } \\
\text { CCMP1375 }\end{array}$ & Unicellular Marine & 1883 & - & - & - \\
\hline Prochlorococcus marinus str. MIT 9211 & Unicellular Marine & 1855 & - & - & - \\
\hline Prochlorococcus marinus str. MIT 9313 & Unicellular Marine & 2269 & - & - & - \\
\hline Prochlorococcus marinus str. MIT 9303 & Unicellular Marine & 2997 & - & - & - \\
\hline Synechococcus sp. WH 7803 & Unicellular Marine & 2533 & - & - & - \\
\hline Synechococcus sp. CC 9311 & Unicellular Marine & 2892 & - & - & - \\
\hline Synechococcus sp. WH 8102 & Unicellular Marine & 2519 & - & - & - \\
\hline Synechococcus sp. CC 9902 & Unicellular Marine & 2307 & - & - & - \\
\hline Synechococcus sp. CC 9605 & Unicellular Marine & 2645 & - & - & - \\
\hline Synechococcus sp. RCC 307 & Unicellular Marine & 2535 & - & - & - \\
\hline Synechococcus elongatus PCC 6301 & Unicellular Freshwater & 2527 & $1(0.04 \%)$ & 1 & - \\
\hline Synechococcus elongatus PCC 7942 & Unicellular Freshwater & 2612 & $1(0.04 \%)$ & 1 & - \\
\hline Acaryochloris marina MBIC 11017 & Unicellular Symbiont & 6254 & $8(0.13 \%)$ & 1 & 29 \\
\hline Microcystis aeruginosa NIES-843 & Unicellular Freshwater & 6312 & $2(0.03 \%)$ & 1 & - \\
\hline Synechocystis sp. PCC 6803 & Unicellular Freshwater & 3172 & $1(0.03 \%)$ & 1 & - \\
\hline Cyanothece sp. ATCC 51142 & Unicellular Marine & 4762 & $3(0.06 \%)$ & 2 & 16 \\
\hline Synechococcus sp. PCC 7002 & Unicellular Marine & 2823 & $1(0.04 \%)$ & 1 & - \\
\hline Trichodesmium erythraeum IMS 101 & Filamentous Nonheterocystous & 4451 & $10(0.22 \%)$ & 1 & 62 \\
\hline Nostoc punctiforme PCC 73102 & $\begin{array}{l}\text { Filamentous Heterocystous } \\
\text { Symbiotic }\end{array}$ & 6087 & $9(0.15 \%)$ & 2 & 6 \\
\hline Nostoc sp. PCC 7120 & $\begin{array}{l}\text { Filamentous Heterocystous } \\
\text { Freshwater }\end{array}$ & 5366 & $7(0.13 \%)$ & 2 & 43 \\
\hline Anabaena variabilis ATCC 29413 & Filamentous Heterocystous Soil & 5043 & $9(0.18 \%)$ & 2 & 64 \\
\hline Gloeobacter violaceus PCC 7421 & Unicellular Rock & 4430 & $4(0.09 \%)$ & - & 56 \\
\hline Synechococcus sp. JA-2-3B'a(2-13) & Unicellular Hot spring & 2862 & $1(0.03 \%)$ & 1 & - \\
\hline Synechococcus sp. JA-3-3Ab & Unicellular Hot spring & 2760 & $1(0.04 \%)$ & 1 & - \\
\hline
\end{tabular}

hypothetical proteins, and 2 proteins annotated as unknown function protein DUF323.

Amid diverse cyanobacterial genomes, the number of metacaspase genes varies from 0 to 10 . Within unicellular cyanobacteria, Symbiont Acaryochloris marina MBIC 11017 has 8 MCAs, much more than other species. Correspondingly, the percentage of metacaspases within total proteins $(0.13 \%)$ is highest among unicellular cyanobacteria. All of the marine Synechococcus strains lack MCAs except for Synechococcus sp. PCC 7002. Only one metacaspase gene was found in the Synechocystis sp. PCC 6803. Four Synechococcus strains inhabit in freshwater, land and hot spring contain one metacaspase for each, including PCC 6301, PCC 7942, JA-2-3B'a(2-3), and JA-3-3Ab. Water-blooming cyanobacterium Microcystis aeruginosa NIES-843 contains two MCAs. The percentages of MCAs within total proteins $(0.03 \%$ $-0.04 \%)$ in Synechocystis sp., Synechococcus sp. and Microcystis sp. strains are the lowest. The other two unicellular cyanobacterial strains, Gloeobacter violaceus PCC 7421 and Cyanothece sp. ATCC 51142, maintain 3 to 4 MCAs and have moderate percentages of MCAs in total proteins: $0.09 \%$ and $0.06 \%$, respectively.

Compared to unicellular cyanobacteria, filamentous diazotrophic cyanobacteria have more metacaspase genes (10 for Trichodesmium erythraeum IMS 101, 9 for Anabaena variabilis ATCC 29413, 9 for Nostoc punctiforme PCC 73102, and 7 for Nostoc sp. PCC 7120). 


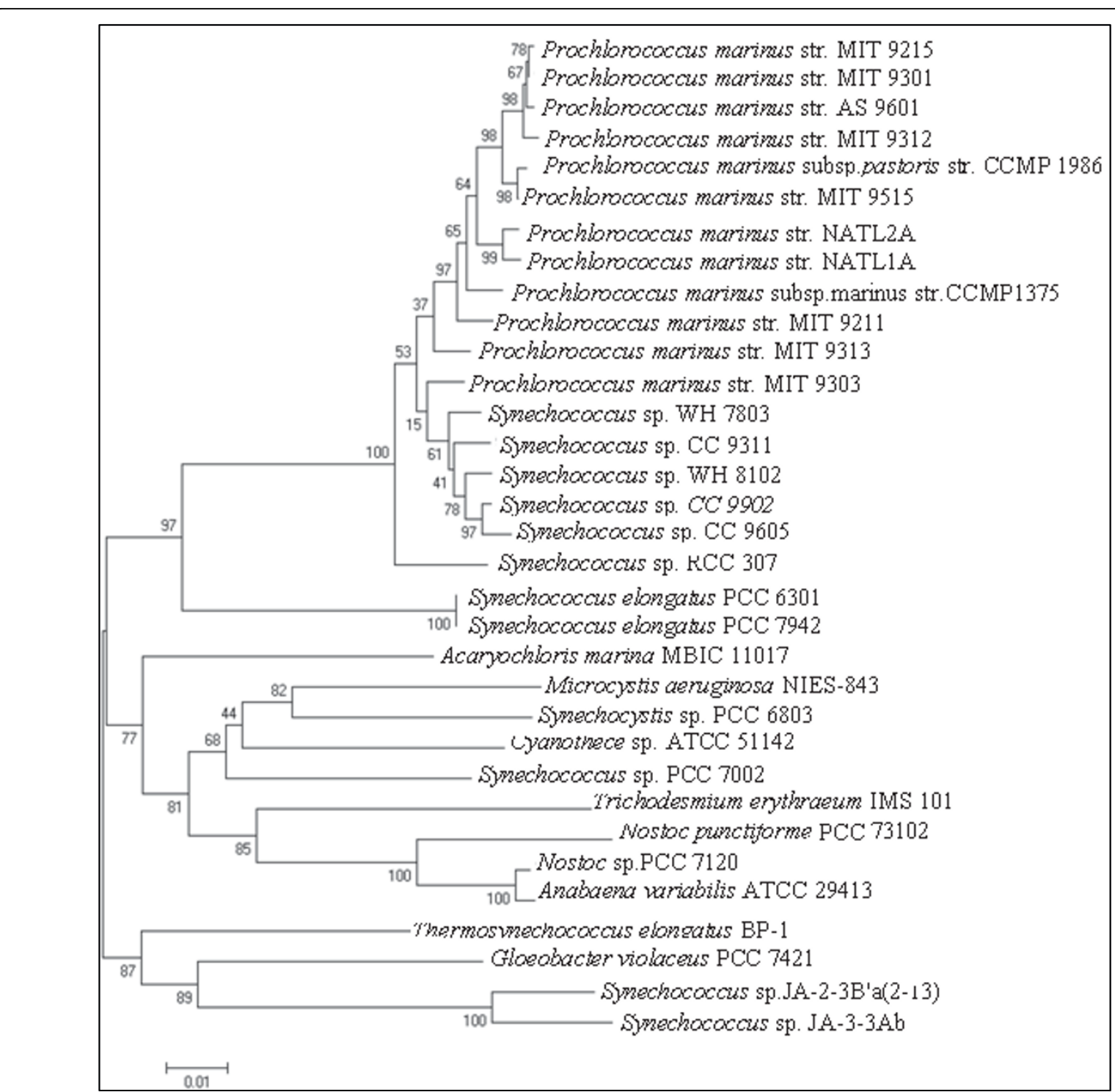

Figure 1 Phylogenetic tree of the sequenced cyanobacterial strains based on 16s rRNA. Phylogenetic tree reconstruction of the 33 fully sequenced cyanobacteria was performed based on 16s rRNA as described in the Methods section. The number on each branch indicates a bootstrap probability (1000 replicates).

Among the filamentous cyanobacteria, Nostoc punctiforme PCC 73102 has the largest genome; however, nonheterocystous cyanobacterium Trichodesmium erythraeum IMS 101 contains the largest number of metacaspases and the highest percentage in total protein (0.22\%) (Table 1).

One or two MCAs containing mutations of conserved catalytic sites (His/Cys) were found in every cyanobacterial strain, exclusive of Gloeobacter violaceus PCC 7421. In most cases, the His residues are replaced by Tyr, and the Cys residues mutate into Ser, Asn or Gly. Uniquely, within the metacaspase (641678142) from Cyanothece sp. ATTC 51142, Gln substitutes for the Cys residue (Table 1, 2).

\section{Structure and function}

Based on structural characteristics, we classify the identified cyanobacterial metacaspases into two families, $\operatorname{cbMC} \alpha$ and $\operatorname{cbMC} \beta$ (Figure 2).

Cyanobacterial metacaspase Family $\alpha(\operatorname{cbMC} \alpha)$ includes 27 MCAs that process none other identifiable domains than P20. This family was further divided into four subfamilies. Subfamily $\alpha$ ( $\operatorname{cbMC} \alpha-\mathrm{I})$ processing the CASc domain only, contains one MCA (637235425 from Nostoc $s p$. PCC 7120). Subfamily II (cbMC $\alpha-\mathrm{TM})$ maintaining a single transmembrane (TM) domain, contains five MCAs found in filamentous cyanobacteria Anabaena variabilis ATCC 29413, Nostoc punctiforme PCC 73102, Nostoc sp. PCC 7120, and unicellular 
Table 2 Cyanobacterial putative metacaspase gene

\begin{tabular}{|c|c|c|c|c|}
\hline Gene $^{a}$ & Family & His/Cys Sites & Additional Domians & Annotation \\
\hline \multicolumn{5}{|c|}{ Acaryochlor is marina MBIC 11017} \\
\hline 641253645 & $\mathrm{cbMC} \alpha$-other & $H-Y C-G$ & & Protease (caspase) p20 domain containing protein \\
\hline 641252580 & $\mathrm{cbMC} \beta$ & & NACHT, WD40(13) & WD-40 repeat protein \\
\hline 641249149 & $\mathrm{cbMC} \beta$ & & WD40(7) & WD-repeat protein \\
\hline 641257459 & $\mathrm{cbMC} \beta$ & & Pentapeptide(4) & Peptidase C14, caspase catalytic subunit p20 \\
\hline 641250651 & $\mathrm{cbMC} \beta$ & & GUN4 & GUN4-like family protein \\
\hline 641254504 & $\operatorname{cbMC} \alpha-T M$ & & & WD-40 repeat protein \\
\hline 641249463 & $\mathrm{cbMC} \beta$ & & DUF323 & Hypothetical protein \\
\hline 641257535 & $\mathrm{cbMC} \beta$ & & DEXDC, HELICC & Dead/death box helicase domain protein \\
\hline \multicolumn{5}{|c|}{ Anabaena variabilis ATCC 29413} \\
\hline 637717727 & $\mathrm{cbMC} \alpha$-other & H-Y C-S & & Peptidase C14, caspase catalytic subunit p20 \\
\hline 637718597 & $\operatorname{cbMC} \alpha$-other & & & Peptidase $\mathrm{C} 14$, caspase catalytic subunit p20 \\
\hline 637715366 & $\mathrm{cbMC} \beta$ & & WD40(14) & Peptidase C14, caspase catalytic subunit p20 \\
\hline 637719457 & $\mathrm{cbMC} \alpha$-other & & & Peptidase $C 14$, caspase catalytic subunit p20 \\
\hline 637717526 & $\mathrm{cbMC} \beta$ & & WD40(14) & Peptidase $C 14$, caspase catalytic subunit p20 \\
\hline 637717527 & $\mathrm{cbMC} \beta$ & & WD40(8) & Peptidase C14, caspase catalytic subunit p20 \\
\hline 637718424 & $\mathrm{cbMC} \beta$ & & WD40(14) & Peptidase $\mathrm{C} 14$, caspase catalytic subunit p20 \\
\hline 637718423 & $\mathrm{cbMC} \beta$ & & WD40(14) & Peptidase C14, caspase catalytic subunit p20 \\
\hline 637718962 & $\operatorname{cbMC} \alpha-\mathrm{TM}$ & $\mathrm{H}-\mathrm{Y}$ C-N & & Peptidase $\mathrm{C} 14$, caspase catalytic subunit p20 \\
\hline \multicolumn{5}{|c|}{ Cyanothece sp. ATTC 51142} \\
\hline 641679166 & cbMC $\alpha$-other & $\mathrm{H}-\mathrm{Y}$ C-S & & Putative peptidase $\mathrm{C} 14$, caspase catalytic \\
\hline 641676675 & $\mathrm{cbMC} \beta$ & & NACHT, WD40(15) & WD-40 repeat protein \\
\hline 641678142 & $\operatorname{cbMC} \alpha$-other & $\mathrm{H}-\mathrm{Y}$ C-Q & & Putative peptidase $\mathrm{C} 14$, caspase catalytic \\
\hline \multicolumn{5}{|c|}{ Gloeobacter violaceus PCC 7421} \\
\hline 637459639 & $\mathrm{cbMC} \beta$ & & WD40(14) & WD-repeat protein \\
\hline 637458101 & $\mathrm{cbMC} \beta$ & & WD40(14) & WD-40 repeat protein \\
\hline 637459020 & $\mathrm{cbMC} \beta$ & & WD40(14) & WD-40 repeat protein \\
\hline 637461074 & $\mathrm{cbMC} \beta$ & & WD40(14) & WD-40 repeat protein \\
\hline \multicolumn{5}{|c|}{ Microcystis aeruginosa NIES-843 } \\
\hline 641536480 & cbMc $\alpha$-other & & & Peptidase C14, caspase catalytic subunit p20 \\
\hline 641535722 & $\mathrm{cbMC} \alpha$-other & H-Y C-S & & Peptidase C14, caspase catalytic subunit p20 \\
\hline \multicolumn{5}{|c|}{ Nostoc punctiforme PCC 73102} \\
\hline 638389336 & $\mathrm{cbMC} \alpha$-other & & & Hypothetical protein \\
\hline 638391264 & cbMC $\alpha$-other & & & Hypothetical protein \\
\hline 638390474 & $\operatorname{cbMC} \alpha$-other & H-Y C-S & & Hypothetical protein \\
\hline 638392408 & $\mathrm{cbMC} \alpha-\mathrm{TM}$ & & & Hypothetical protein \\
\hline 638386606 & $\mathrm{cbMC} \beta$ & & ANF-receptor & $\begin{array}{l}\text { ABC-type branched-chain amino acid transport systems, } \\
\text { periplasmic component }\end{array}$ \\
\hline 638389051 & $\mathrm{cbMC} \beta$ & & DUF323 & Chromosome segregation ATPases \\
\hline 638388760 & $\mathrm{cbMC} \beta$ & & Pentapeptide(3) & Uncharacterized protein containing caspase domain \\
\hline 638390427 & $\operatorname{cbMC} \alpha-\mathrm{TM}$ & $\mathrm{H}-\mathrm{Y}$ C-N & & Uncharacterized protein containing caspase domain \\
\hline 638392333 & $\mathrm{cbMC} \beta$ & & GUN4 & Uncharacterized protein containing caspase domain \\
\hline \multicolumn{5}{|c|}{ Nostoc sp. PCC 7120} \\
\hline 637235546 & cbMc $\alpha$-other & $\mathrm{H}-\mathrm{Y} C-\mathrm{S}$ & & Hypothetical protein \\
\hline 637230642 & $\mathrm{cbMC} \beta$ & & WD40(14) & WD-40 repeat protein \\
\hline 637230643 & $\mathrm{cbMC} \beta$ & & WD40(14) & WD-40 repeat protein \\
\hline 637232504 & $\mathrm{cbMC} \beta$ & & WD40(14) & WD-40 repeat protein \\
\hline 637235425 & $\mathrm{cbMC} \alpha$ & & & Hypothetical protein \\
\hline 637233614 & $\mathrm{cbMC} \beta$ & & DUF323 & Hypothetical protein \\
\hline 637234068 & $\mathrm{cbMC} \alpha-\mathrm{TM}$ & $\mathrm{H}-\mathrm{Y}$ C-N & & Hypothetical protein \\
\hline \multicolumn{5}{|c|}{ Synechococcus elongatus PCC 6301} \\
\hline 637616879 & cbMC $\alpha$-other & C-G & & Hypothetical protein \\
\hline
\end{tabular}


Table 2: Cyanobacterial putative metacaspase gene (Continued)

\begin{tabular}{|c|c|c|c|c|}
\hline \multicolumn{5}{|c|}{ Synechococcus elongatus PCC 7942} \\
\hline 637798702 & cbMC $\alpha$-other & $C-G$ & & Hypothetical protein \\
\hline \multicolumn{5}{|c|}{ Synechococcus sp. JA-2-3B'a(2-13) } \\
\hline 637875026 & cbMC $\alpha$-other & $\mathrm{H}-\mathrm{Y} C-\mathrm{G}$ & & Peptidase, C14 family \\
\hline \multicolumn{5}{|c|}{ Synechococcus sp. JA-3-3Ab } \\
\hline 637872245 & cbMC $\alpha$-other & $H-Y C-G$ & & ICE-like protease (caspase) p20 domain protein \\
\hline \multicolumn{5}{|c|}{ Synechococcus sp. PCC 7002} \\
\hline 641610111 & cbMC $\alpha$-other & $\mathrm{H}-\mathrm{Y} \mathrm{C}-\mathrm{S}$ & & ICE-like protease (caspase) p20 domain protein \\
\hline \multicolumn{5}{|c|}{ Synechocystis sp. PCC 6803} \\
\hline 637011435 & cbMC $\alpha$-other & $\mathrm{H}-\mathrm{Y} C-\mathrm{G}$ & & Hypothetical protein \\
\hline \multicolumn{5}{|c|}{ Trichodesmium erythraeum IMS 101} \\
\hline 638107693 & cbMc $\alpha$-other & $\mathrm{H}-\mathrm{Y}$ C-S & & Peptidase $\mathrm{C} 14$, caspase catalytic subunit p20 \\
\hline 638106962 & $\mathrm{cbMC} \beta$ & & EZ-HEAT(15) & Peptidase $\mathrm{C} 14$, caspase catalytic subunit p20 \\
\hline 638107555 & $\mathrm{cbMC} \beta$ & & WD40(14) & Peptidase $\mathrm{C} 14$, caspase catalytic subunit p20 \\
\hline 638109494 & $\mathrm{cbMC} \beta$ & & WD40(15) & WD-40 repeat \\
\hline 638108799 & $\mathrm{cbMC} \beta$ & & WD40(15) & WD-40 repeat \\
\hline 638107819 & $\mathrm{cbMC} \alpha-\mathrm{C}$ & & & Peptidase $C 14$, caspase catalytic subunit p20 \\
\hline 638107188 & $\mathrm{cbMC} \beta$ & & DUF323 & Protein of unknown function DUF 323 \\
\hline 638107169 & cbMC $\alpha$-other & & & Peptidase $\mathrm{C} 14$, caspase catalytic subunit p20 \\
\hline 638107752 & $\mathrm{cbMC} \beta$ & & DUF323 & Protein of unknown function DUF 323 \\
\hline 638105709 & $\operatorname{cbMC} \beta$ & & CHASE2 & Putative Chase2 sensor protein \\
\hline
\end{tabular}

a: the numbers indicate "IMG Gene Object Identifier" in IMG database

cyanobacterium Acaryochloris marina MBIC 11017. Subfamily III (cbMC $\alpha$-other) contains 20 proteins, which is the majority in cyanobacterial MCAs. These MCAs have an unidentified C-terminal domain each, and distribute among all cyanobacterial strains except Gloeobacter violaceus PCC 7421. MCAs attributed to subfamily I to III process CASc in their N-terminal. While the single MCA of Subfamily IV (cbMC $\alpha-C)$, 638107819 from Trichodesmium erythraeum ISM 101, holds the CASc domain in the C-terminal.

Cyanobacterial metacaspase Family $\beta$ (cbMC $\beta$ ) occupying at least one additional domain, comprises 31 (53.4\%) metacaspases from all filamentous cyanobacteria and three unicellular species (Cyanothece sp. ATCC 51142, Acaryochloris marina MBIC 11017, and Gloeobacter violaceus PCC 7421). The number of additional domains varies from 16 to 56. Gloeobacter violaceus PCC 7421 contains the largest number of additional domains, which is 14 times than that of the total MCAs (Table 1). Seven unicellular strains lack additional domains, including Synechococcus sp. strains, Microcystis aeruginosa NIES-843 and Synechocystis sp. PCC 6803.

In total, 10 types of additional domains were identified in cyanobacterial MCAs: ANF-receptor, WD40, GUN4, NACHT, DUF323, CHASE2, Pentapeptide, DEXDc, HELICc, and EZ-HEAT (Figure 2, Table 3). Most of these domains are involved in signal transduction, for example CHASE2, GUN4, ANF-receptor and WD40 (Table 3). In addition, prevalent domains with scaffolding or unknown functions in bacteria were identified, such as DUF323, EZ HEAT and Pentapeptide [21]. Two domains within helicases, HELICc and DEXDc, were found to fuse together in 641257535 of Acaryochloris marina MBIC 11017. Interestingly, PCD related domain NACHT was also detected in two MCAs (641676675 from Cyanothece sp. ATCC 51142 and 641252580 from Acaryochloris marina MBIC 11017).

WD40 repeats, the most prevalent additional domains identified in 6 cyanobacterial species, replicate for 7 to 15 copies in a single metacaspase protein. Some additional domains were identified exclusively in a particular metacaspase protein, including DEXDc and HELICc in 641257535 (Acaryochloris marina MBIC 11017); EZ HEAT in 638106962 (Trichodesmium erythraeum ISM 101), CHASE2 in 638105709 (Trichodesmium erythraeum ISM 101), and ANF-receptor in 638386606 (Nostoc punctiforme PCC 73102).

\section{Phylogenetic analysis}

Considering the confusion created by additional domains with possibly separate evolutionary histories, the conserved catalytic domains of MCAs instead of their whole sequences were used during the phylogenetic study (Figure 3 ). The catalytic domains, about 340 amino acids in length, were identified using SMART and CDD databases $[23,26]$. The MCA phylogenetic tree was rooted in the human caspase- 3 and the putative metacaspase of Gamma proteobacterium. 


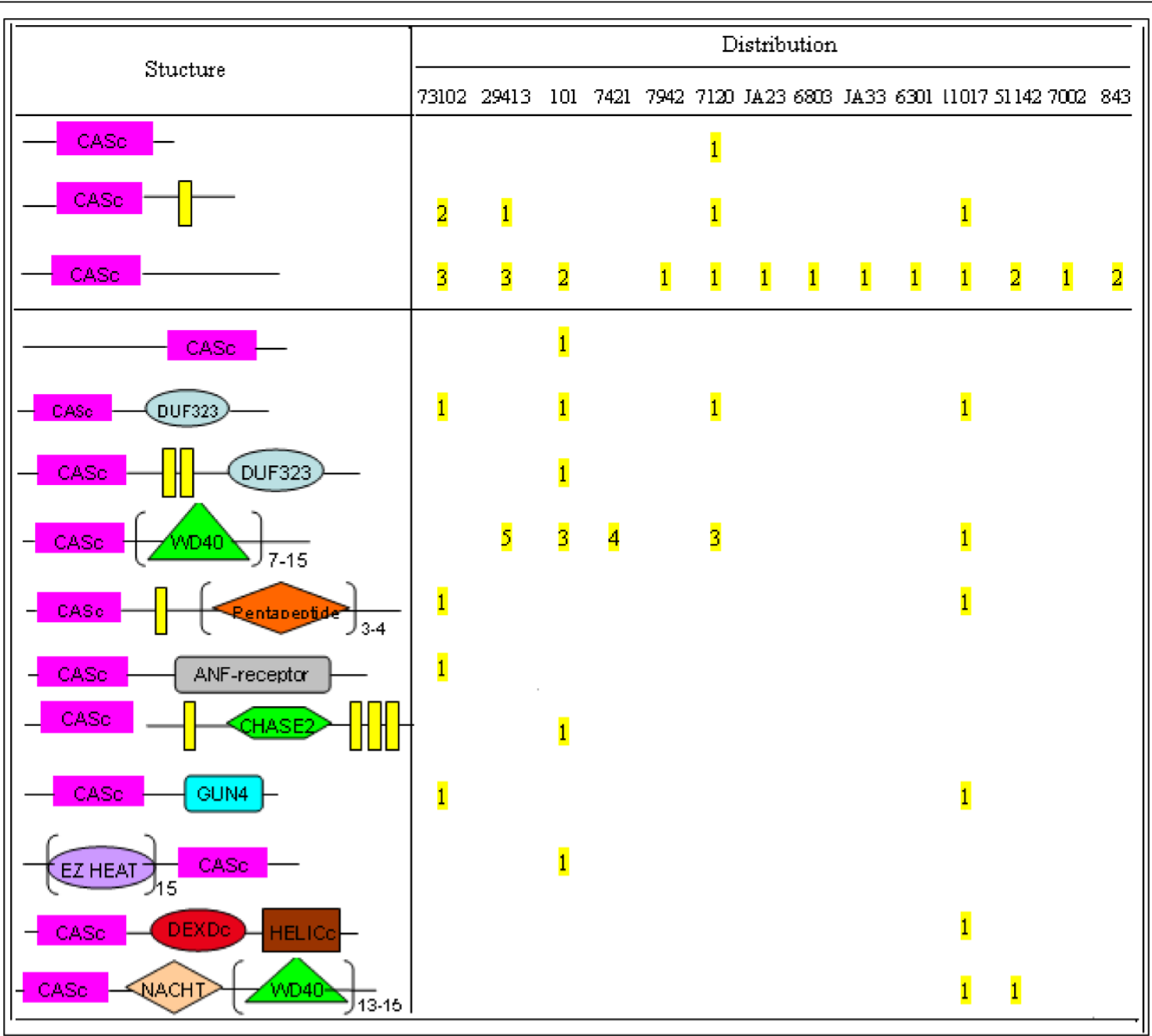

Figure 2 Domain organization and distribution of putative cyanobacterial metacaspases. Fused domains that form a single polypeptide chain are connected by a horizontal line. The yellow rectangles represent transmembrane (TM) domain. Strain and domain names are as in Table 1 and 3, respectively. Figures are not drawn to scale.

Table 3 Additional domains of cyanobacterial MCA Gene

\begin{tabular}{|c|c|c|c|}
\hline Abbreviation & Domain & Functions & Distribution \\
\hline ANF-receptor & ANF-receptor & extracellular ligand binding domain & $\begin{array}{l}\text { transmembrane receptors (such as histidine kinases, } \\
\text { serine/threonine kinases) }\end{array}$ \\
\hline CHASE2 & CHASE2 & $\begin{array}{l}\text { extracellular sensory in signal } \\
\text { transduction }\end{array}$ & $\begin{array}{l}\text { transmembrane receptors (such as histidine kinases, } \\
\text { serine/threonine kinases) }\end{array}$ \\
\hline DEXDC & $\begin{array}{l}\text { DEAD-like helicases } \\
\text { superfamily }\end{array}$ & ATP-binding & $\begin{array}{l}\text { proteins involved in ATP-dependent RNA or } \\
\text { DNA unwinding }\end{array}$ \\
\hline DUF323 & Domain of unknown function & unknown & bacterial unknown proteins \\
\hline EZ HEAT & E-Z type HEAT repeats & scaffold & cyanobacterial phycocyanin lyase and other proteins \\
\hline GUN4 & GUN4-like domain & $\begin{array}{l}\text { signaling; accumulation of glycolipids } \\
\text { into the heterocysts }\end{array}$ & cyanobacterial serine/threonine kinases \\
\hline NACHT & NACHT domain & programmed cell death & apoptotic proteins \\
\hline WD40 & WD40 domain & regulator in signal transduction & cyanobacterial serine/threonine kinases \\
\hline pentapeptide & $\begin{array}{l}\text { pentapeptide } \\
\text { repeats }\end{array}$ & $\begin{array}{l}\text { accumulation of glycolipids into } \\
\text { the heterocysts }\end{array}$ & unicellular and filamentous cyanobacterial proteins \\
\hline
\end{tabular}


The phylogeny tree comprises two clades in general. MCAs from cbMC $\alpha$ belong to Clade I (green box in Figure 3) that also includes two bacterial MCAs (637773422 from Chlorobium chlorochromatii and 642380481 from Rhizobium leguminosarum) and seven eukaryotic MCAs from T. pseudonana, S. cerevisiae and Chlamydomonas reinhardtii. All of these MCAs are orthologues because of obvious evolutionary relationships with high bootstrap value. Clade II (grey box in Figure 3) contains members of cbMC $\beta$ family with WD40, DUF323 and signalling domains, which cluster separately according to the additional domains. Those from subfamily cbMC $\alpha$-TM and eubacteria (Rhizobium leguminosarum and Rhodopseudomonas palustris) attribute to Clade II as well. In a word, most photosynthetic bacterial MCAs cluster with MCAs of cyanobacterial family $\beta$ and the eukaryotic MCAs gather with family $\alpha$. Within each clade, the MCAs cluster according to the phylogeny of the species.

Within clade I, one copy of MCA containing the His/ Cys mutation is found in each cyanobacterium that maintains MCA, except for Gloeobacter violaceus PCC 7421. All of these MCAs belong to cbMC $\alpha$-other and cluster strictly based on the phylogeny of the species. All of the mutated MCAs in clade I are orthologs because of their close evolutionary relationships. Besides, four mutated MCAs from one unicellular and three filamentous cyanobacteria gather together in clade II.

Anabaena variabilis ATCC 29413 and Nostoc sp. PCC 7120 , two filamentous species that show close evolutionary relationships in $16 \mathrm{~S}$ rRNA tree, share 3 pairs of MCA sequences in clade I. Four WD40-containing MCAs from Gloeobacter violaceus PCC 7421 form a separate cluster together. In addition, some MCAs adjacent to each other on the chromosome display close evolutionary relationships, including 637717526/ 637717527, 637718423/637718424 of Anabaena variabilis ATCC 29413 and 637230642/637230643 of Nostoc sp. PCC 7120. Some MCAs of subfamily $\alpha$ are flanked with two WD40- or GUN4-containing MCAs.

\section{Discussion}

Although metacaspases do not cleave caspase substrates $[12,18,27-29]$, several evidences have been given to support their roles in PCD in plants (see review [30]). For example, when challenged by the plant pathogen, Arabidopsis $\mathrm{KO}$ lines of metacaspase suppress cell death [31]. Arabidopsis metacaspse- $8 \mathrm{KO}$ lines triggered by UVC or $\mathrm{H}_{2} \mathrm{O}_{2}$ display reduced cell death [12]. Moreover, in the suspensor cells of an embryogenic culture of Picea abies, down-regulation of MCA leads to a phenotype with a reduced cell death [32].

What makes metacaspases so interesting? First, most of the metazoan PCD-related genes are lost in 
unicellular organisms, excepting metacaspases that play vital roles in PCD of eukaryotic planktons and yeasts. Second, compared with caspases and paracaspases identified in higher animals, metacaspases are widespread among bacteria, fungi and plants, which suggest their early evolutionary positions [33].

Bidle and Falkowski identified cyanobacterial and phytoplankton metacaspases in silico, and explored the evolution deeply [20]. With the completion of genome sequencing of several cyanobacterial species, modifications and supplements are needed. For example, MCA was reported to be absent in Synechococcus sp. PCC 7942, but a protein (ID: 637798702, annotation: hypothetical protein) was found to contain P20 domain. Cyanobacterium Thermosynechococcus elongatus BP-1 was proved to have no metacaspase orthologue in our study. Moreover, with the release of genomic sequence, MCAs were identified in Synechococcus sp. JA-3-3Ab and Synechococcus sp. JA-2-3B'a(2-13).

The distribution of putative metacaspase encoding open readingframes (ORFs) in cyanobacteria is an integrated function of the genome sizes and the ecophysiological properties. Most cyanobacteria process proportionate numbers of putative metacaspase genes with genome sizes, except for symbiotic Acaryochloris marina MBIC 11017. Though death is not the only way to adapt to environmental changes, for example, cyanobacteria modify their metabolism in response to different stress conditions [34], death is still a direct and drastic cellular response to environmental changes. Thus diverse distributions of metacaspase genes may reflect various environmental selective pressures. For example, putative MCA encoding ORFs are not widespread through unicellular cyanobacteria (Table 1). All of the Prochlorococcus and Synechococcus strains lived in the oligotrophic open ocean lack putative metacaspase genes. While Synechococcus strains that inhabit in freshwater and hot spring still maintain one metacaspase encoding ORF. Considering the similar genome size, environmental selective pressure may take responsibility for this difference. Parallel conclusion was provided by Serine/threonine kinases in cyanobacteria indicating remarkable reduction of signal transduction proteins and environmental stress response systems in the ocean [21]. Gene lost is revealed to facilitate these cyanobacteria to acclimatize to the oligotrophic environment. The major driving force was supposed to be "a selective process favouring the adaptation of these cyanobacteria", which was discussed by Alexis Dufresne et al. in detail [35]. Filamentous heterocystous cyanobacteria, on the other hand, differentiate heterocysts in response to the absence of combined nitrogen, and exhibit ecological properties including broad symbiotic competence with plants and fungi [21], contain more putative MCA encoding ORFs even after allowing for their larger genome sizes [36].
The symbol of caspase superfamily is the possession of catalytic P20 domain and the conserved Cys-His dyad, forming the "specificity pocket" [9]. Within cyanobacterial metacaspases, sequence contexts of His and Cys are basically the same as those in caspases (His:(Y/F)SGHG, and Cys:QAC(R/Q)G) [17]. The maintaining of the conserved His and Cys indicate the importance of these catalytic sites. Interestingly, 17 cyanobacterial MCAs encode Tyr in place of His and Ser/Asn/Gln/Gly instead of Cys. Likewise, of two metacaspases in T. brucei, TbMCA1 and TbMCA4, Ser occupies the site of the putative Cys [37]. Considering the fact that experimental mutation of the active-site cysteine to serine resulted in inactive of some cysteine peptidases, mutated cyanobacterial metacaspases may be catalytically inactive.

Domain fusion provides a chance to recruit related functions in a single protein, especially within bacteria which maintain smaller genomes and compact gene clusters [19]. Additional domains of cyanobacterial MCAs, such as GUN4 [38] and WD40 [39], illuminate the signalling pathways involved in PCD. Owe to the considerably specific proteolytic activity and proximity-induced activation, the signalling domains may be the target of the metacaspases. Consequently, metacaspases may take a share in signalling mediation instead of mere protein degradation. Two domains within helicases, HELICc and DEXDc, were found to fuse together in 641257535 of Acaryochloris marina MBIC 11017, which implies the interactions between the caspase proteolytic activity and ATP-dependent RNA/ DNA unwinding. In addition, domains with same functions tend to assemble in a protein [19], therefore the identification of NACHT $[19,22,40]$ domain reinforces the possibility of metacaspase involving in PCD. Compared with metazoan caspases, bacterial metacaspases may present a minimal set of apoptotic machinery. Additional domains are typically employed as "sensor response modules" and form multi-domain proteins with MCAs to participate in signal transduction. It can be imagined that caspases recruit additional domains or even large motifs to apoptotic complexes in the evolution.

Previous classification criterion of MCAs is based on the prodomain, and MCAs can be classified into two families "Type I with an N-terminal extension" and "Type II with a linker region between the putative large and small subunits") $[9,18,36]$. However, most cyanobacterial MCAs maintain a C-terminal extension without the linker region and have varied additional domains (Figure 2, Figure 3). Therefore a novel categorization standard based on the additional domain was given in this research. To avoid future confusion of the MCA families, the names "Type $\alpha$ " and "Type $\beta$ " were used instead of "Type I" and "Type II".

The obtained metacaspase phylogenetic tree indicates that MCAs display clear-cut relationships, based strictly 
on structural characteristics and the phylogeny of the species. Moreover, compared to the species phylogeny, structural characteristics play a more critical role. The tree of catalytic P20 domains coincides well with the phylogenies based on 16s rRNA, indicating the rare gene gain-and-loss events and the importance of MCAs that remain conserved in history. Besides, four WD40containing MCAs from Gloeobacter violaceus PCC 7421 form a separate cluster indicating obvious lineage-specific duplication events. Anabaena variabilis ATCC 29413 and Nostoc sp. PCC 7120, two filamentous species that enjoy a very close evolutionary relatedness in 16S rRNA tree, share 3 pairs of MCA sequences in clade I. These nonorthologous MCAs may be produced by gene duplication before the divergence of the two species.

Most photosynthetic bacterial MCAs cluster with MCAs of cyanobacterial family $\beta$ (with additional domain) and the eukaryotic MCAs cluster with family $\alpha$ (without additional domain). The presence of the mutated MCAs and their convergences suggest that MCAs of family $\alpha$ and $\beta$ evolve separately. One possibility is that the recruitment of WD40 additional domain occurs later than the divergence of the two clades.

\section{Conclusions}

The availability of cyanobacterial genome sequences facilitates comparative analysis. Metacaspases, sequence homologs to caspases, play key roles in programmed cell death (PCD) in several prokaryotes, fungi and plants. Among 33 species of cyanobacteria, a total of 58 putative metacaspase genes have been identified. The quantity of metacaspase genes in unicellular and filamentous cyanobacteria depends on the genome size and ecological habitat. The Cys-His dyad of caspase superfamily is conserved in most cyanobacterial MCAs, however, Tyr and Ser/Asn/Gln/Gly residues have also been detected in the sites of His and Cys within some metacaspases. Ten types and a total of 276 additional domains were identified, most of which may involve in signal recognition. Programmed cell death related NACHT domain was also found in cyanobacterial metacaspases. Phylogenetic tree of MCA catalytic P20 domains coincides well with the phylogenies based on 16s rRNA.

\section{Methods}

Thirty-three species of cyanobacteria, including Prochlorococcus, Synechococcus, Synechocystis, Gloeobacter, Cyanothece, Microcystis, Trichodesmium, Acaryochloris, Anabaena and Nostoc were used in this analysis. Since sequences of 36 species had not been fully released, they were not considered in our comparisons. All of the 33 genome sequences (as of Nov. 2008) were accessed from IMG in FASTA format [41].
In order to identify genes that may encode metacaspases, proven metacaspases from marine diatom Thalassiosira pseudonana (Protein id: 270038, 2505, 268857, 270007, 38187 in Thalassiosira pseudonana "finished chromosomes" database v3.0 [11,42]) were used to construct a query protein set. BLASTp (protein-protein BLAST) $[22,43,44]$ was conducted locally to search all proteins from each of the 33 cyanobacteria. Proteins found by this method that fit the criteria for a genuine metacaspase were added to the query set for another round of BLASTp searches. A threshold e-value of 1e10 was set in the first two rounds, which changed into $2 \mathrm{e}-20$ subsequently. The procedure was continued until no new proteins were found.

Proteins identified by BLASTp were aligned using Clustal X (Version 1.83) program [45] with a gap opening penalty of 10 , a gap extension penalty of 0.2 , and Gonnet as the weight matrix. The alignment was examined by inspection of peptidase $\mathrm{C} 14$, caspase catalytic subunit P20 domain (COG 4249, KOG1546 in the NCBI Conserved Domain Database [23,24]). A protein was accepted as a metacaspase if it was possible to recognize P20 domain and if the most conserved His and Cys residues known to participate in the function of metacaspases [18] were present. However, minor alterations of the conserved His and Cys residues were tolerated. Specifically, putative MCA genes encoding Tyr in place of His and Ser/Asn/Gln/Gly instead of Cys were taken into account as well. Structure analyses of the obtained metacaspases were performed using the SMART (Simple Modular Architecture Research Tool) [25,26] and the CDD (Conserved Domains Database) [23,24], relying on hidden Markov models and Reverse Position-Specific BLAST separately. Sequences of the P20 domain (about 300 aa in length) used for phylogenetic tree construction were obtained from the SMART database $[25,26]$. Trees based on metacaspase P20 domain and cyanobacterial 16s rRNA were constructed using NJ methods of the MEGA package (Version 4.0) [46], and the reliability of each branch was tested by 1000 bootstrap replications. In phylogenetic analysis of MCA, putative metacaspase of Gamma proteobacterium and human caspase-3 were used as outgroups to root the tree.

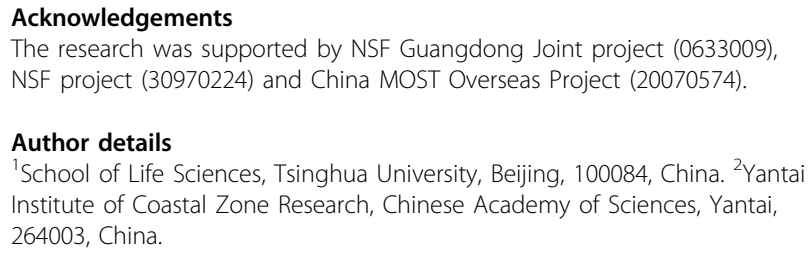

${ }^{1}$ School of Life Sciences, Tsinghua University, Beijing, 100084, China. ${ }^{2}$ Yantai Institute of Coastal Zone Research, Chinese Academy of Sciences, Yantai, 264003, China.

\section{Authors' contributions}

Qiao Jiang conceived of the study, participated in the sequence analysis, and drafted the manuscript. Song Qin and Qing-yu Wu participated in its 
coordination and helped to draft the manuscript. All authors read and approved the final manuscript.

Received: 8 September 2009 Accepted: 25 March 2010

Published: 25 March 2010

\section{References}

1. Stanier RY, Cohen-Bazire G: Phototrophic prokaryotes: the cyanobacteria. Annu Rev Microbiol 1977, 31:225-274.

2. Partensky F, Hess WR, Vaulot D: Prochlorococcus, a marine photosynthetic prokaryote of global significance. Microbiol Mol Biol Rev 1999, 63(1):106-127.

3. Duque-Parra JE: Note on the origin and history of the term "apoptosis". Anat Rec B New Anat 2005, 283(1):2-4.

4. F.Madeo EF, Frohlich $\mathrm{KU}$ : A yeast mutant showing diagnostic markers of early and late apoptosis. J Cell Biol 1997, 139:729-734.

5. Deponte M: Programmed cell death in protists. Biochim Biophys Acto 2008, 1783(7):1396-1405.

6. Ning SB, Guo HL, Wang L, Song YC: Salt stress induces programmed cell death in prokaryotic organism Anabaena. J Appl Microbiol 2002, 93(1):15-28.

7. Ross C, Santiago-Vazquez L, Paul V: Toxin release in response to oxidative stress and programmed cell death in the cyanobacterium Microcystis aeruginosa. Aquat Toxicol 2006, 78(1):66-73.

8. Berman-Frank I, Rosenberg G, Levitan O, Haramaty L, Mari X: Coupling between autocatalytic cell death and transparent exopolymeric particle production in the marine cyanobacterium Trichodesmium. Environ Microbiol 2007, 9(6):1415-1422.

9. Uren AG, O'Rourke K, Aravind LA, Pisabarro MT, Seshagiri S, Koonin EV Dixit VM: Identification of paracaspases and metacaspases: two ancient families of caspase-like proteins, one of which plays a key role in MALT lymphoma. Mol Cell 2000, 6(4):961-967.

10. Khan MA, Chock PB, Stadtman ER: Knockout of caspase-like gene, YCA1, abrogates apoptosis and elevates oxidized proteins in Saccharomyces cerevisiae. Proc Natl Acad Sci USA 2005, 102(48):17326-17331.

11. Bidle KD, Bender SJ: Iron starvation and culture age activate metacaspases and programmed cell death in the marine diatom Thalassiosira pseudonana. Eukaryot Cell 2008, 7(2):223-236.

12. He R, Drury GE, Rotari VI, Gordon A, Willer M, Farzaneh T, Woltering EJ, Gallois P: Metacaspase- 8 modulates programmed cell death induced by ultraviolet light and $\mathrm{H} 2 \mathrm{O} 2$ in Arabidopsis. J Biol Chem 2008, 283(2):774-783.

13. Lim HW, Kim SJ, Park EH, Lim CJ: Overexpression of a metacaspase gene stimulates cell growth and stress response in Schizosaccharomyces pombe. Can J Microbiol 2007, 53(8):1016-1023.

14. Le Chat L, Sinden RE, Dessens JT: The role of metacaspase 1 in Plasmodium berghei development and apoptosis. Mol Biochem Parasitol 2007, 153(1):41-47.

15. Trzyna WC, Legras XD, Cordingley JS: A type-1 metacaspase from Acanthamoeba castellanii. Microbiol Res 2008, 163(4):414-423.

16. Bidle KD, Haramaty L, Barcelos ERJ, Falkowski P: Viral activation and recruitment of metacaspases in the unicellular coccolithophore, Emiliania huxleyi. Proc Natl Acad Sci USA 2007, 104(14):6049-6054

17. Mottram JC, Helms MJ, Coombs GH, Sajid M: Clan CD cysteine peptidases of parasitic protozoa. Trends Parasitol 2003, 19(4):182-187.

18. Vercammen D, Cotte van de B, De Jaeger G, Eeckhout D, Casteels P, Vandepoele K, Vandenberghe I, Van Beeumen J, Inze D, Van Breusegem F: Type II metacaspases Atmc4 and Atmc9 of Arabidopsis thaliana cleave substrates after arginine and lysine. J Biol Chem 2004, 279(44):45329-45336.

19. Koonin EV, Aravind L: Origin and evolution of eukaryotic apoptosis: the bacterial connection. Cell Death Differ 2002, 9(4):394-404.

20. Bidle KD, Falkowski PG: Cell death in planktonic, photosynthetic microorganisms. Nat Rev Microbiol 2004, 2(8):643-655.

21. Zhang X, Zhao F, Guan X, Yang Y, Liang C, Qin S: Genome-wide survey of putative serine/threonine protein kinases in cyanobacteria. BMC Genomics 2007, 8:395.

22. Fedorova ND, Badger JH, Robson GD, Wortman JR, Nierman WC: Comparative analysis of programmed cell death pathways in filamentous fungi. BMC Genomics 2005, 6:177.
23. Marchler-Bauer A, Bryant SH: CD-Search: protein domain annotations on the fly. Nucleic Acids Res 2004, 32 Web Server: W327-331.

24. NCBI Conserved Domain Database (CDD). [http://www.ncbi.nlm.nih.gov/ structure/cdd/cdd.shtml].

25. SMART Home. [http://smart.embl-heidelberg.de/].

26. Schultz J, Milpetz F, Bork P, Ponting CP: SMART, a simple modular architecture research tool: identification of signaling domains. Proc Nat Acad Sci USA 1998, 95(11):5857-5864.

27. Bozhkov PV, Suarez MF, Filonova LH, Daniel G, Zamyatnin AA Jr, RodriguezNieto S, Zhivotovsky B, Smertenko A: Cysteine protease mcll-Pa executes programmed cell death during plant embryogenesis. Proc Natl Acad Sci USA 2005, 102(40):14463-14468

28. Watanabe N, Lam E: Two Arabidopsis metacaspases AtMCP1b and AtMCP2b are arginine/lysine-specific cysteine proteases and activate apoptosis-like cell death in yeast. J Biol Chem 2005, 280(15):14691-14699.

29. Vercammen D, Belenghi B, Cotte van de B, Beunens T, Gavigan JA, De Rycke R, Brackenier A, Inze D, Harris JL, Van Breusegem F: Serpin1 of Arabidopsis thaliana is a suicide inhibitor for metacaspase 9. J Mol Biol 2006, 364(4):625-636

30. Bonneau L, Ge Y, Drury GE, Gallois P: What happened to plant caspases? J Exp Bot 2008, 59(3):491-499.

31. Van Baarlen PWE, Staats M, Van Kan JAL: Histochemical and genetic analysis of host and non-host interactions of Arabidopsis with three Botrytis species: an important role for cell death control. Molecular Plant Pathology 2007, 8:41-54.

32. Suarez MF, Filonova LH, Smertenko A, Savenkov El, Clapham DH, von Arnold S, Zhivotovsky B, Bozhkov PV: Metacaspase-dependent programmed cell death is essential for plant embryogenesis. Curr Biol 2004, 14(9):R339-340.

33. Boyce M, Degterev A, Yuan J: Caspases: an ancient cellular sword of Damocles. Cell Death Differ 2004, 11(1):29-37.

34. Castielli O, De la Cerda B, Navarro JA, Hervas M, De la Rosa MA: Proteomic analyses of the response of cyanobacteria to different stress conditions. FEBS Lett 2009, 583(11):1753-1758.

35. Dufresne A, Garczarek L, Partensky F: Accelerated evolution associated with genome reduction in a free-living prokaryote. Genome Biol 2005, 6(2):R14.

36. Vercammen D, Declercq W, Vandenabeele P, Van Breusegem F: Are metacaspases caspases? J Cell Biol 2007, 179(3):375-380.

37. Szallies A, Kubata BK, Duszenko M: A metacaspase of Trypanosoma brucei causes loss of respiration competence and clonal death in the yeast Saccharomyces cerevisiae. FEBS Lett 2002, 517(1-3):144-150.

38. Larkin RM, Alonso JM, Ecker JR, Chory J: GUN4, a regulator of chlorophyl synthesis and intracellular signaling. Science 2003, 299(5608):902-906.

39. Smith TF: Diversity of WD-repeat proteins. Subcell Biochem 2008, 48:20-30.

40. Koonin EV, Aravind L: The NACHT family - a new group of predicted NTPases implicated in apoptosis and MHC transcription activation. Trends Biochem Sci 2000, 25(5):223-224.

41. IMG Home. [http://img.jgi.doe.gov/cgi-bin/pub/main.cgi]

42. Thalassiosira pseudonana "finished chromosomes" database v3.0. [http:// genome.jgi-psf.org/Thaps3/Thaps3.home.html].

43. Altschul SF, Gish W, Miller W, Myers EW, Lipman DJ: Basic local alignment search tool. J Mol Biol 1990, 215(3):403-410.

44. Altschul SF, Madden TL, Schaffer AA, Zhang J, Zhang Z, Miller W, Lipman DJ: Gapped BLAST and PSI-BLAST: a new generation of protein database search programs. Nucleic Acids Res 1997, 25(17):3389-3402.

45. Larkin MA, Blackshields G, Brown NP, Chenna R, McGettigan PA, McWilliam H, Valentin F, Wallace IM, Wilm A, Lopez R, et al: Clustal W and Clustal X version 2.0. Bioinformatics 2007, 23(21):2947-2948.

46. Tamura K, Dudley J, Nei M, Kumar S: MEGA4: Molecular Evolutionary Genetics Analysis (MEGA) software version 4.0. Mol Biol Evol 2007, 24(8):1596-1599.

doi:10.1186/1471-2164-11-198

Cite this article as: Jiang et al.: Genome-wide comparative analysis of metacaspases in unicellular and filamentous cyanobacteria. BMC Genomics 2010 11:198. 